\title{
Implementation of co-trimoxazole preventive therapy policy for malaria in HIV-infected pregnant women in the public health facilities in Tanzania
}

\section{Appolinary AR Kamuhabwa Richard Gordian Ritah F Mutagonda}

Department of Clinical Pharmacy and Pharmacology, School of Pharmacy, Muhimbili University of Health and Allied Sciences, Dar es Salaam, Tanzania
Correspondence: Appolinary AR

Kamuhabwa

Department of Clinical Pharmacy and

Pharmacology, School of Pharmacy,

Muhimbili University of Health and Allied

Sciences, PO Box 65013, Dar es Salaam,

Tanzania

Tel +25575 5576985

Fax +255222150465

Email enali2012@gmail.com
This article was published in the following Dove Press journal:

Drug, Healthcare and Patient Safety

13 December 2016

Number of times this article has been viewed

Background: In 2011, Tanzania adopted a policy for provision of daily co-trimoxazole prophylaxis to HIV-infected pregnant women for prevention of malaria and other opportunistic infections. As per the policy, HIV-infected pregnant women should not be given sulfadoxinepyrimethamine (SP) for intermittent preventive therapy. The challenges associated with this policy change and the extent to which the new policy for prevention of malaria in pregnant women coinfected with HIV was implemented need to be assessed.

Aim: To assess the implementation of malaria-preventive therapy policy among HIV-infected pregnant women in the public health facilities in Dar es Salaam, Tanzania.

Methodology: The study was conducted in Kinondoni Municipality, Dar es Salaam, Tanzania, from January 2015 to July 2015. Three hundred and fifty-three HIV-infected pregnant women who were attending antenatal clinics (ANCs) and using co-trimoxazole for prevention of malaria were interviewed. Twenty-six health care workers working at the ANCs were also interviewed regarding provision of co-trimoxazole prophylaxis to pregnant women. A knowledge scale was used to grade the level of knowledge of health care providers. Focus group discussions were also conducted with 18 health care workers to assess the level of implementation of the policy and the challenges encountered.

Results: Twenty-three (6.5\%) pregnant women with known HIV serostatus were using cotrimoxazole for prevention of opportunistic infections even before they became pregnant. Out of the $353 \mathrm{HIV}$-infected pregnant women, eight $(2.5 \%)$ were coadministered with both SP and co-trimoxazole. Sixty (16.7\%) pregnant women had poor adherence to co-trimoxazole prophylaxis. Out of the 26 interviewed health care providers, 20 had high level of knowledge regarding malaria-preventive therapy in HIV-infected pregnant women. Lack of adequate supply of co-trimoxazole in health facilities and inadequate training of health care providers were among the factors causing poor implementation of co-trimoxazole prophylaxis for prevention of malaria in HIV-infected pregnant women.

Conclusion: There is a need to continue sensitization of pregnant women and communities about the importance of early attendance to the ANCs for testing of HIV and provision of cotrimoxazole prophylaxis. Availability of co-trimoxazole in the health facilities, regular training, and sensitization of health care providers are necessary for effective implementation of this policy. Keywords: SP, policy change, ANCs, health care providers, IPTp

\section{Introduction}

Malaria infection during pregnancy has been reported to have adverse effects including maternal anemia, low birth weight (LBW), intrauterine growth retardation, intrauterine 
death, stillbirth, premature delivery, and perinatal and neonatal morbidity and mortality. ${ }^{1,2}$ Therefore, early diagnosis and effective case management of malaria in pregnant women are crucial in preventing progression of uncomplicated malaria to severe disease and death. Moreover, coinfection of malaria with HIV further complicates pregnancies leading to increased maternal morbidity and negative birth outcomes. ${ }^{3-5}$

A number of interventions have been implemented to reduce prevalence of malaria in Tanzania. The use of insecticide-treated nets (ITNs), intermittent preventive therapy using sulfadoxine-pyrimethamine (IPTp-SP), and effective malaria case management are among the strategies that have been used to prevent and manage malaria in pregnant women. The combination of these strategies has successfully reduced the prevalence of malaria from $18.1 \%$ in 2008 to $9.5 \%$ in 2012. However, the prevalence of malaria has increased to $14.8 \%$ in 2016 . This trend is reported to be due to decrease in the ITNs coverage from $56 \%$ in $2011 / 2012$ to $39 \%$ in $2015 / 2016$. Also, the use of ITNs has declined from $75 \%$ in $2011 / 2012$ to $54 \%$ in 2016 among pregnant women. There is therefore a need to ensure continued implementation of these strategies for effective prevention of malaria and its negative outcomes in pregnant women. ${ }^{6-8}$

In HIV-infected pregnant women, malaria parasite density is increased compared to non-HIV-infected pregnant women. As a result, HIV-infected pregnant women have reduced response to antimalarial treatment, leading to poor birth outcomes such as fetal loss, preterm delivery, and LBW. ${ }^{1}$ The total estimated number of HIV-infected pregnant women as per the Tanzania PMTCT report is $122,146 .{ }^{9}$ The percentage of infants born to HIV-infected mothers who become HIV infected is about $15 \% .{ }^{10}$ Children born to women with HIV and malaria have LBW and are more likely to die during infancy. ${ }^{11}$ Malaria enhances suppression of body immunity and therefore facilitates progression of HIV infection to AIDS. Therefore, infants born to HIV-infected mothers not taking antiretroviral drugs (ARVs) have significantly higher risk (35-40\% chance) of mother-to-child transmission of HIV during breastfeeding. ${ }^{12}$

In order to reduce the negative effects of malaria and HIV coinfection, the World Health Organization recommends pregnant women in malaria-endemic areas to sleep under insecticide-treated bed nets, to use IPTp, to be treated with effective and safe antimalarial drugs, and to use iron supplements for prevention of anemia during the second and third trimesters. ${ }^{3}$ In Tanzania, like other African countries, monthly IPTp-SP is recommended at antenatal clinics (ANCs). ${ }^{13}$ For HIV-infected pregnant women, universal co-trimoxazole prophylaxis is recommended to prevent opportunistic infections including malaria regardless of CD4 count in malariaendemic areas. ${ }^{14}$

Two randomized trials conducted in Uganda and Malawi reported co-trimoxazole prophylaxis to be non-inferior to IPTp with respect to infant mortality, LBW $(<2.5 \mathrm{~kg})$, placental malaria, maternal death, and treatment-limiting adverse events. ${ }^{15}$ Concomitant use of co-trimoxazole and sulfadoxine-pyrimethamine (SP), especially in HIV-infected individuals, is contraindicated because of increased risk of severe cutaneous reactions. ${ }^{16,17}$ Therefore, HIV-infected pregnant women who are already receiving co-trimoxazole prophylaxis should not receive IPTp-SP. ${ }^{18}$

In Tanzania, the policy for provision of daily co-trimoxazole prophylaxis to HIV-infected pregnant women was adopted in the year 2011. ${ }^{19}$ Currently, there are no published reports on how effectively has the policy been implemented in the health facilities in Tanzania. In the implementation of this policy, a number of issues need to be addressed. These include training of health care providers, ensuring constant availability of co-trimoxazole tablets in health facilities, sensitization of pregnant women, and integration of maternal and child health care services in health facilities. ${ }^{20,21}$

With support from development partners, Tanzania has been able to effectively roll out provision of ARV therapy countrywide. There have also been efforts to integrate antenatal services to ensure comprehensive care for pregnant women in the ANCs. ${ }^{22}$ Pregnant women attending ANCs are counseled, tested for HIV, and provided with prevention of mother-to-child HIV transmission (PMTCT) services. Late booking and lack of completion of scheduled visits to ANCs are likely the main challenges for early identification of HIVinfected pregnant women for initiation of co-trimoxazole prophylaxis and other antenatal services. ${ }^{13}$

Therefore, continuous sensitization of communities and pregnant women about the importance of antenatal care is necessary for effective implementation of co-trimoxazole prophylaxis in HIV-infected pregnant women. Understanding the level of implementation of this policy and challenges encountered is essential in order to institute the necessary interventions. In this study, the level of the implementation of co-trimoxazole prophylaxis in HIV-infected pregnant women in the public health facilities in the urban area of Dar es Salaam was assessed. The focus was on HIV-infected pregnant women who were attending ANCs for routine care and co-trimoxazole prophylaxis. Health care workers in the ANCs were also interviewed in order to get their views regarding this policy and its level of implementation. 


\section{Methods}

\section{Study design and sites}

This was a descriptive cross-sectional study which employed both quantitative and qualitative research methods. This study design was chosen in order to assess the level of implementation of co-trimoxazole prophylaxis at the health facilities in Dar es Salaam at that given time. In this study, HIV-infected pregnant women and health care providers at the ANCs were interviewed, patient files were reviewed, and focus group discussions (FGDs) were conducted with health care providers. The study was conducted at the ANCs in three public health facilities in Kinondoni Municipality in Dar es Salaam, Tanzania. These facilities were Mwananyamala Regional Referral Hospital, Sinza Hospital, and Magomeni Health Centre. The study was conducted from January to July 2015.

\section{Sample size determination}

Study population included HIV-infected pregnant women and health care workers at the ANCs. Sample size for HIVinfected pregnant women was estimated using a statistical formula for cross-sectional studies based on the study done in Tanzania in $2011 .{ }^{23}$ In that study, the prevalence of HIV among women who were attending ANCs was 5.6\%. In total, 353 HIV-infected pregnant women were recruited and interviewed when attending ANCs. In addition, files of HIVinfected pregnant women who were attending ANCs for IPTp with co-trimoxazole were reviewed.

Due to the shortage of health care workers in the health facilities, especially in the ANCs, the sample size for health care providers was not calculated. Therefore, all health care providers (pharmacists, medical doctors, clinical officers, and nurses) working at the ANCs, especially in the PMTCT units, who were present during the time of the study were included in the study. FGDs were conducted with health care providers to get information about the implementation of co-trimoxazole prophylaxis policy in HIV-infected pregnant women in the health facilities.

Participants for inclusion in this study were HIV-infected pregnant women who were attending ANCs for antenatal care and IPTp for malaria, and health care providers working at ANCs mainly in PMTCT units. Severely ill HIV-infected pregnant women who were admitted were not included in the study.

\section{Data collection}

\section{Interviews of pregnant women}

Structured questionnaires containing closed- and open-ended questions were used to interview and generate data from HIVinfected pregnant women attending ANCs. Information from files of HIV-infected pregnant women was obtained using a designed form. This form was designed specifically to collect data for this study. The focus was on the use of co-trimoxazole prophylaxis for prevention of malaria in HIV-infected pregnant women. Questions were mainly based on understanding of the necessity for early attendance to the ANCs, awareness about the benefits of the use of co-trimoxazole prophylaxis during pregnancy, and dosage and duration of use of co-trimoxazole.

\section{Interviews of health care providers}

Self-administered questionnaires were filled by the health care providers working at the ANCs. A knowledge scale was prepared consisting of closed-ended questions; one point was awarded for each correct answer and a zero point for a wrong answer. The knowledge level of health care providers was then graded as low (0-1), medium (2-3), and high (4-5) depending on the questions in the check list.

Health care workers were asked about the availability of guidelines for PMTCT at ANCs, if the language used in the guidelines is easily understandable, and the use of co-trimoxazole prophylaxis for prevention of malaria in HIV-infected pregnant women. The questions were based on the level of understanding of the use of co-trimoxazole in HIV-infected pregnant women, dosage of co-trimoxazole required for prophylaxis, and implementation of the policy.

\section{Focus group discussions}

FGDs were conducted by a social scientist and researchers using prepared guided format containing probes based on the objectives of the study. FGDs were conducted at ANCs at Mwananyamala Regional Referral Hospital and Magomeni Health Centre. A total of 18 health care providers participated in the FGDs. There were at least eight participants in each focus group. Discussions were recorded and later on translated to address the objectives of the study.

\section{Health facility assessment}

The main purpose of this assessment was to identify the level of implementation of the malaria-preventive therapy policy in HIV-infected pregnant women at the health facilities and the challenges encountered. Facility assessment form was used to assess the availability of essential resources (trained human resources, guidelines, co-trimoxazole tablets, malaria and HIV diagnostic test kits).

\section{Data analysis}

Data obtained using questionnaires and data collection forms were coded. The coded data were entered in the computer for 
analysis using the Statistical Package for Social Sciences program version 20 and Microsoft Excel. Data were compared using chi-square test. Results were considered statistically significant at a $p$-value of $<0.05$. Fischer's exact test was used to test for association between the knowledge of health care providers and the study variables.

Discussion and comments recorded during FGDs were transcribed, and then the main points for each question were documented. Quotations which illustrated each theme were identified.

\section{Ethical approval}

Ethical approval for the study was sought from Muhimbili University of Health and Allied Sciences, Research and Publications Committee. Permission to conduct the study at the ANCs in the public health facilities was granted from Kinondoni Municipal Council-District Medical Officer. Before being interviewed, participants were provided with information about the study, and a written informed consent was obtained. In order to ensure confidentiality of study participants, data were entered in the computer and analyzed using code numbers instead of names.

\section{Results}

\section{Interviews of HIV-infected pregnant women regarding provision of co- trimoxazole prophylaxis in the ANCs}

Out of the 353 HIV-infected pregnant women who were recruited in the study, $153(43.3 \%)$ were in the second trimester, and $200(56.7 \%)$ were in the third trimester of pregnancy. Sixty-four (18.1\%) pregnant women were primigravidae (first pregnancy), 128 (36.3\%) were secundigravidae (second pregnancy), and 161 (45.6\%) were multigravidae who have had three or more pregnancies at the time of interviews. Twenty-four $(6.8 \%)$ pregnant women were in the age group of 17-20 years, $126(35.7 \%)$ were in the age group of 20-30 years, and $155(44.0 \%)$ were aged $31-40$ years. Majority (81.6\%) of pregnant women had attained primary level of education, and $64 \%$ were employed in the formal sector (Table 1).

All $353 \mathrm{HIV}$-infected pregnant women mentioned that they were counseled and were prescribed with co-trimoxazole after receiving their HIV results. Provision of co-trimoxazole is done in special units called "PMTCT units" in the health facilities. All of the interviewed pregnant women said that cotrimoxazole tablets are given for free in the health facilities. During interviews, it was revealed that 23 (6.5\%) pregnant women were already using co-trimoxazole for prevention of
Table I Sociodemographic characteristics of HIV-infected pregnant women who were interviewed in the health facilities $(n=353)$

\begin{tabular}{|c|c|c|}
\hline $\begin{array}{l}\text { Sociodemographic } \\
\text { characteristics }\end{array}$ & $\begin{array}{l}\text { Number of } \\
\text { respondents }\end{array}$ & Percentage \\
\hline \multicolumn{3}{|l|}{ Age group (years) } \\
\hline $17-20$ & 24 & 06.8 \\
\hline $20-30$ & 126 & 35.7 \\
\hline $31-40$ & 155 & 44.0 \\
\hline$>41$ & 48 & 13.5 \\
\hline \multicolumn{3}{|l|}{ Education level } \\
\hline Primary & 288 & 81.6 \\
\hline Secondary & 47 & 13.3 \\
\hline College/university level & 18 & 5.1 \\
\hline \multicolumn{3}{|l|}{ Marital status } \\
\hline Married & 203 & 57.5 \\
\hline Not married & 59 & 16.7 \\
\hline Cohabitating & 80 & 22.7 \\
\hline Divorced & 8 & 2.3 \\
\hline Widowed & 3 & 0.85 \\
\hline \multicolumn{3}{|l|}{ Current trimester } \\
\hline Second & 153 & 43.3 \\
\hline Third & 200 & 56.7 \\
\hline \multicolumn{3}{|l|}{ Gravida } \\
\hline Primigravidae & 64 & 18.1 \\
\hline Secundigravidae & 128 & 36.3 \\
\hline $\begin{array}{l}\text { Multigravidae (three or more } \\
\text { pregnancies) }\end{array}$ & 161 & 45.6 \\
\hline \multicolumn{3}{|l|}{ Occupation } \\
\hline Housewife & 67 & 19.0 \\
\hline Employed in formal sector & 226 & 64.0 \\
\hline Self-employed & 63 & 17.8 \\
\hline
\end{tabular}

opportunistic infections even before they became pregnant because of their known HIV serostatus.

At the PMTCT units, all HIV-infected pregnant women were given monthly doses of co-trimoxazole of two singlestrength tablets (sulfamethoxazole $80 \mathrm{mg}$ and trimethoprim $400 \mathrm{mg}$ ) to be taken on a daily basis. Most (85\%) of the pregnant women mentioned that they take co-trimoxazole tablets after dinner in the night. The main reason for taking the tablets at night was the perceived co-trimoxazole-induced side effects. Five (1.4\%) HIV-infected pregnant women had stopped to take co-trimoxazole due to severe cutaneous reactions.

Out of 353 HIV-infected pregnant women, eight (2.5\%) were coadministered with both SP and co-trimoxazole. Out of the eight patients who were coadministered with SP and cotrimoxazole, five were attending ANCs at Magomeni Health Centre and three at Sinza Hospital. These pregnant women were given SP during their first antenatal visit before testing for HIV. After testing for HIV, they were given co-trimoxazole as well. These were pregnant women who attended the clinics with pregnancy of $>14$ weeks. It was found out that this was mainly caused by lack of communication between health care workers in PMTCT unit and other units in the ANCs. 
During interviews, pregnant women who were coadministered with SP and co-trimoxazole during their first visit were given three tablets of SP which were taken as direct observed therapy. After confirming that they were HIV-infected, they were directed to PMTCT units. At the PMTCT units, health care providers gave the pregnant women monthly doses of co-trimoxazole tablets to be taken at home. Interviews with pregnant women at Mwananyamala hospital revealed that pregnant women in that health facility are not given any prophylactic treatment during the first visit until confirmation of their serostatus.

\section{Self-reported adherence to co-trimoxazole prophylaxis among pregnant women}

Out of 353 HIV-infected pregnant women interviewed at the three ANCs, 59 (16.7\%) had poor adherence to co-trimoxazole prophylaxis. Out of those with poor adherence, 27 (45.8\%) women mentioned that they had missed at least two doses of co-trimoxazole prophylaxis for the past one month (ie, since last visit to the ANCs). On the other hand, nine $(15.3 \%)$ pregnant women mentioned that they had missed more than three doses of co-trimoxazole prophylaxis for the past one month (Table 2).

During the interviews, pregnant women were asked about the reasons for non-adherence to co-trimoxazole prophylaxis. Twenty-two (37.3\%) out of the 59 non-adherent pregnant women mentioned co-trimoxazole-induced side effects, including nausea, vomiting, and skin rashes, as the reasons for skipping prescribed doses. Seventeen (28.8\%) pregnant women mentioned stigma to HIV infection as the main reason for non-adherence. This is especially caused by lack of privacy at home for taking the medicines because some pregnant women had not disclosed their HIV serostatus to family members. Travels outside Dar es Salaam and social events such as attending parties, marriage, or burial ceremonies were mentioned by $12(30.3 \%)$ pregnant women as causes of nonadherence to co-trimoxazole prophylaxis. Eight (13.6\%) pregnant women mentioned concomitant use of co-trimoxazole with other medications, especially ARV drugs, as one of the reasons for skipping doses of co-trimoxazole prophylaxis.

\section{Interviews of health care providers regarding provision of co-trimoxazole prophylaxis in the ANCs}

Sociodemographic characteristics of health care providers who were interviewed at the ANCs are presented in Table 3. Out of 26 health care providers, 25 mentioned that they have
Table 2 Frequency of not taking co-trimoxazole prophylaxis during the past one month as reported by pregnant women $(n=59)$

\begin{tabular}{lll}
\hline $\begin{array}{l}\text { Number of times in } \\
\text { which doses were missed }\end{array}$ & $\begin{array}{l}\text { Number of } \\
\text { pregnant women }\end{array}$ & Percentage \\
\hline Once & 9 & 15.3 \\
Twice & 27 & 45.8 \\
Thrice & 14 & 23.7 \\
More than thrice & 9 & 15.3 \\
\hline
\end{tabular}

Table 3 Sociodemographic characteristics of health care providers who were interviewed at the antenatal clinics $(n=26)$

\begin{tabular}{lll}
\hline $\begin{array}{l}\text { Sociodemographic } \\
\text { characteristics }\end{array}$ & $\begin{array}{l}\text { Number of } \\
\text { respondents }\end{array}$ & Percentage \\
\hline Sex & 5 & 19.2 \\
Male & 21 & 80.8 \\
Female & & \\
Age group (years) & 7 & 27.0 \\
20-30 & 10 & 38.5 \\
$31-40$ & 6 & 23.1 \\
$41-50$ & 3 & 11.4 \\
$51-60$ & & \\
Work experience (years) & 14 & 53.8 \\
$<10$ & 5 & 19.2 \\
I0-20 & 7 & 27.0 \\
>20 & & \\
Marital status & 20 & 77.0 \\
Married & 5 & 19.2 \\
Not married & 1 & 3.8 \\
Divorced & & \\
Designation & 3 & 11.5 \\
Medical doctor & 1 & 3.8 \\
Clinical officer & 3 & 11.5 \\
Pharmacist & 3 & 11.5 \\
Registered nurse & 6 & 23.1 \\
Nurse officer & 3 & 11.5 \\
Assistant nurse officer & 4 & 15.4 \\
Enrolled nurse & 3 & 11.5 \\
Nurse assistant & &
\end{tabular}

documented adherence strategies for co-trimoxazole prophylaxis at their ANCs. Fifteen health care providers said that one of the major strategies to ensure that HIV-infected pregnant women adhere to co-trimoxazole prophylaxis is to provide them with continuing health education during every visit to the ANC. Education is based on malaria prevention methods and the importance of co-trimoxazole prophylaxis for prevention of malaria and other opportunistic infections. Other mentioned strategies were ensuring availability of co-trimoxazole tablets at the health facilities. Pill count was mentioned as one of the strategies to enhance adherence when pregnant women return to the ANCs for routine check-up or prescription refill. It was mentioned by health care providers that pill count is not a reliable means of assessing adherence because some pregnant women discard some tablets or share with family members 
prior to visiting the ANCs. During the FGDs, one registered nurse at Mwananyamala Regional Referral Hospital said:

Apart from continuous health education we also remind pregnant women about the scheduled dates to attend ANCs. Reminder is done through phone calls, as part of the strategy to enhance adherence to antiretroviral drugs and other medications including co-trimoxazole prophylaxis. Home-based care approach is another strategy in which HIV-infected pregnant who have already consented to disclose their HIV status are visited at home for counseling to enhance adherence to drugs and attendance to ANCs.

Twenty-three health care providers mentioned that cotrimoxazole-induced side effects were the main cause of non-adherence to the drug. Stigma to HIV and lack of health information were other factors mentioned by health care workers to be the cause of non-adherence to co-trimoxazole prophylaxis in pregnant women. In addition, lack of effective supply chain management leading to frequent stock-outs of co-trimoxazole tablets in the health facilities was reported by 10 health care providers as one of the impediments for provision of co-trimoxazole prophylaxis. Lack of communication among health care workers working in PMTCT and other units in the health facilities was also mentioned as a cause for not providing co-trimoxazole prophylaxis in accordance to the guidelines. During the FGDs, one of the participants at Mwananyamala Regional Referral Hospital said:

\footnotetext{
Another factor which contributes to non-adherence to cotrimoxazole prophylaxis in $\mathrm{HIV}$-infected pregnant women is spiritual belief, especially born-again Christians who stop taking medications through advice by religious leaders and their own beliefs. In these churches, pregnant women are encouraged to pray with belief that God will cure them through prayers. An example was cited in which the husband of one of the HIV-infected pregnant woman reported to the ANC that his wife had stopped taking antiretroviral drugs and co-trimoxazole after started going to one of the local born-again Christian Church.
}

Continuous provision of health education to pregnant women at every ANC visit was reported by majority of health care providers as the factor which increases the rate of adherence to co-trimoxazole prophylaxis among HIV-infected pregnant women (Table 4). During health education sessions at the ANCs, important health information about malaria prevention methods at their homes and the benefits of taking co-trimoxazole prophylaxis during pregnancy is shared. Involvement of one relative to encourage and ensure that
Table 4 Factors which enhance adherence to co-trimoxazole prophylaxis among pregnant women as reported by health care providers in the ANCs $(n=26)$

\begin{tabular}{lll}
\hline Factors & $\begin{array}{l}\text { Number of health } \\
\text { care providers }\end{array}$ & Percentage \\
\hline $\begin{array}{l}\text { Regular provision of health } \\
\text { education at every visit to the }\end{array}$ & 10 & 38.5 \\
$\begin{array}{l}\text { ANC } \\
\text { Emphasis on taking co- }\end{array}$ & 6 & 23.1 \\
trimoxazole during pregnancy & & 15.4 \\
$\begin{array}{l}\text { Ensuring accessibility and } \\
\text { availability of co-trimoxazole } \\
\text { tablets at the ANC }\end{array}$ & 4 & \\
$\begin{array}{l}\text { Involvement of relatives to } \\
\text { encourage adherence } \\
\text { Insisting on visits to scheduled } \\
\text { ANC and regular follow-up }\end{array}$ & 2 & 15.4 \\
\hline
\end{tabular}

Abbreviation: ANC, antenatal clinic.

a pregnant woman takes daily co-trimoxazole prophylaxis increases the rate of adherence among pregnant women.

\section{Level of knowledge of health care providers on malaria prevention policy among HIV-infected pregnant women at ANCs}

Twenty-four out of 26 health care providers mentioned that they have read the malaria prevention policy for HIV-infected pregnant women in Tanzania and national guidelines for PMTCT. They commented that the guidelines are well written and the language used in the guidelines is understandable. They also indicated that they are using these guidelines in the ANCs. Thirteen health care providers said that they had attended special training in relation to malaria-preventive therapy in HIV-infected pregnant women. Twenty-two health care providers mentioned that co-trimoxazole is the drug given to HIV-infected pregnant women for prevention of malaria, while four health care workers mentioned SP.

Twenty-two health care providers who mentioned cotrimoxazole as the approved drug for prevention of malaria in HIV-infected pregnant women knew the correct dose and frequency of use of co-trimoxazole prophylaxis. Regarding the criteria for initiating co-trimoxazole prophylaxis in HIV-infected pregnant women, 15 health care providers said that co-trimoxazole prophylaxis is given to pregnant women who are HIV-positive and with no history of allergic reaction caused by sulfur-containing drugs. Twelve health care workers mentioned that co-trimoxazole prophylaxis should be given to women whose pregnancy is $>14$ weeks and have HIV serostatus confirmed. Twelve health care providers mentioned severe side effects such as skin rashes and Stevens-Johnson 
syndrome as one of the criteria for stopping pregnant women from using co-trimoxazole prophylaxis.

Based on the knowledge scale on implementation of cotrimoxazole prophylaxis in HIV-infected pregnant women, 20 health care providers had high level of knowledge, two had medium, and four health care workers had low level of knowledge. There was an association between the levels of knowledge of health care providers and training on malaria prevention in HIV-infected pregnant women $(p=0.03)$. Out of 20 health care providers who had high level of knowledge on malaria prevention policy among HIV-infected pregnant women, 16 of them reported to have been trained on malaria prevention and treatment in pregnant women.

\section{Health facility assessment}

The main purpose of this assessment was to identify the level of implementation of the malaria-preventive therapy policy at the health facilities and the challenges encountered. Facility assessment revealed that there is lack of pharmaceutical personnel working at the ANCs. Therefore, medicines are dispensed by nurses who are not trained in pharmaceutical management including dispensing. As a result, there was no documentation of strategies for ensuring availability of co-trimoxazole tablets in the health facilities, patient instructions for taking drugs at home, adverse reactions of co-trimoxazole, and information to patients when they experience such effects.

All the assessed health facilities had guidelines for management of HIV-infected pregnant women, including the use of co-trimoxazole prophylaxis in the ANCs. All the health facilities were able to detect malaria infections using malaria rapid diagnostic test. In addition, testing for HIV was routinely done at the ANCs using rapid test kits. There were frequent stock-outs of co-trimoxazole tablets at the PMTCT units. As a result, some pregnant women were required to purchase the drug from private pharmacies. Assessment of patient files indicated that $288(61.2 \%)$ pregnant women started attending ANCs after 20 weeks of gestation.

\section{Discussion}

Before the year 2011, all pregnant women in Tanzania including those infected with HIV were receiving IPTpSP. ${ }^{7,13}$ In 2011, the Government of Tanzania through the ministry responsible for health adopted the policy for provision of daily co-trimoxazole prophylaxis for prevention of malaria and opportunistic infections among HIV-infected pregnant women. ${ }^{19}$ The main objective of this policy was to ensure that all HIV-infected pregnant women are given daily co-trimoxazole prophylaxis throughout the pregnancy to prevent malaria and its negative effects to the mother and unborn baby.

Due to overlapping toxicities between co-trimoxazole and SP, pregnant women receiving co-trimoxazole prophylaxis should not be coadministered with SP. ${ }^{14}$ In this study, all HIV-infected pregnant women attending ANCs were prescribed monthly doses of co-trimoxazole prophylaxis at PMTCT units. However, eight (2.5\%) pregnant women were prescribed both SP and co-trimoxazole. Although guidelines prohibit concomitant use of SP and co-trimoxazole due to overlapping toxicities, ${ }^{24}$ another study also reported the use of the two drugs in HIV-infected pregnant women. ${ }^{25}$ The main reason for concomitant use of these drugs is the lack of knowledge among health care providers in the ANCs. Lack of integration of antenatal care services including IPTp and PMTCT in the health facilities is also a contributing factor for irrational use of SP and co-trimoxazole in HIV-infected pregnant women. ${ }^{26,27}$

In this study, the proportion of self-reported poor adherence to co-trimoxazole prophylaxis was $16.7 \%$. This figure is very low compared to $50.5 \%$ of non-adherent pregnant women which was reported in 2014. ${ }^{28}$ This may indicate improved sensitization of pregnant women to adhere to prescribed co-trimoxazole as a result of integrating antenatal care services in the ANCs. The main reasons for non-adherence in this study were co-trimoxazole-induced side effects, stigma to HIV infection, and patients skipping doses due to attending social events. A study conducted in Tanzania reported increasing prevalence of malaria infection due to non-adherence to co-trimoxazole prophylaxis in pregnant women. ${ }^{28}$ In addition, non-adherence to co-trimoxazole prophylaxis has also been reported to be a contributing factor for increasing resistance of bacteria such as Escherichia coli to most commonly used antibiotics. ${ }^{29}$ Therefore, modifiable factors which have been reported to affect adherence need to be addressed in order to enhance adherence to co-trimoxazole prophylaxis in pregnant women. Occurrence of adverse drug reactions may be one of the reasons for discontinuation of co-trimoxazole prophylaxis in pregnant women. Severe reactions to co-trimoxazole are uncommon, ${ }^{30}$ and in this study, pregnant women reported to have had experienced minor reactions perceived to be due to co-trimoxazole. Cotrimoxazole-induced side effects were reported as the cause of non-adherence to co-trimoxazole prophylaxis. Pregnant women should be provided with information on the potential adverse effects and be counseled to report to the ANC if cotrimoxazole-induced adverse events are suspected. ${ }^{31}$ 
Other factors affecting adherence to co-trimoxazole prophylaxis include concomitant use of ARVs and other medications. Side effects with use of nevirapine include rash and a decrease in white blood cells. These side effects in addition to anemia are frequently seen with the use of co-trimoxazole. ${ }^{32}$ During FGDs, it was mentioned that apart from continuous health education, women are also reminded about the scheduled dates to attend ANCs. Reminder is done through phone calls, as well as home-based care approach, in order to enhance adherence to ARV drugs and other medications including co-trimoxazole prophylaxis. A similar study conducted in Rwanda reported that HIV-infected pregnant women benefit from continuous health education alongside hygiene intervention on malaria prevention during pregnancy. ${ }^{33}$ This study also reported that emphasis on cotrimoxazole prophylaxis during pregnancy is key to enhance the adherence to co-trimoxazole prophylaxis.

Measurement of drug levels in blood is the best method to assess adherence to medications where facilities are available. ${ }^{34}$ Pill counts may underestimate adherence, since some of HIV-infected pregnant women may throw away their pills prior to their clinic visit. Therefore, the level of non-adherence to co-trimoxazole prophylaxis reported in this study may have been underestimated. As observed in this study, rate of adherence can also be enhanced through involvement of close relatives to encourage adherence, ensuring accessibility and availability of co-trimoxazole tablets at ANCs and insisting on attendance to scheduled ANCs. A similar study conducted in Ghana, Kenya, and Malawi, also reported that attendance to ANCs is a key strategy to improve maternal and infant health and also improves adherence to co-trimoxazole prophylaxis. ${ }^{35}$

As reported by majority of health care providers in this study, lack of training on malaria prevention in HIV-infected pregnant women was the main factor causing poor implementation of the policy. There was a positive association between the knowledge level of health care providers and training on malaria prevention in pregnant women. ${ }^{36}$ In order to address impediments for disease control strategies, provision of effective health information and education is necessary. ${ }^{37}$

Frequent stock-out of co-trimoxazole tablets at PMTCT units was reported by health care providers to be one of the reasons for poor implementation of a daily co-trimoxazole prophylaxis in women. For successful provision of co-trimoxazole prophylaxis in pregnant women, it is important to ensure adequate availability of co-trimoxazole in the health facilities. The lack of co-trimoxazole in the health facilities may also be the reason for pregnant women skipping scheduled visits to the ANCs. A recent study conducted in Tanzania reported frequent stock-out of essential medicines including co-trimoxazole in the public health facilities. ${ }^{38}$ The main reason for lack of availability of medicines in these facilities was poor recording resulting in poor quantification of the required medicines. Majority of health care providers in these facilities were not trained pharmaceutical personnel; therefore, they had limited knowledge for pharmaceutical management. As a result, health care providers failed to forecast the required amount of medicines for ordering in the health facilities. ${ }^{39}$ Therefore, emphasis should be made to ensure that inventory management systems for commonly used medicines such as co-trimoxazole are put in place for proper management of medicines in the health facilities.

\section{Conclusion}

The results obtained in this study show that all HIV-infected pregnant women who were attending ANCs and interviewed were provided with co-trimoxazole prophylaxis. The study identified some challenges for effective implementation of co-trimoxazole prophylaxis in HIV-infected pregnant women. These include lack of knowledge among care providers, unavailability of co-trimoxazole tablets in the health facilities, and sensitization of women about the benefits and the need to adhere to treatment. Continued sensitization of pregnant women and communities about the importance of early attendance to the ANCs, availability of co-trimoxazole, and regular training of health care providers are necessary for effective implementation of this policy.

The strength of this study is that different approaches were used including interviews of pregnant women, health care workers, FGDs, and facility assessment. However, due to small number of health care workers in the health facilities, especially in the ANCs, the sample size for health care workers in this study was small and therefore may limit generalization of the findings of this study. Moreover, selfreported adherence to co-trimoxazole prophylaxis has its limitations, and therefore, the proportions of non-adherent pregnant women may have been underestimated. A study with a longer follow-up is recommended to confirm these preliminary results.

\section{Acknowledgments}

The authors acknowledge the financial support provided by the Muhimbili University of Health and Allied Sciences to conduct this study. They thank the management of the Mwananyamala municipal health facilities for granting permission to conduct the study in the facilities. They are grateful to the 
medical officers in charge and other health care workers in the ANCs for providing access and support during interviews and facility assessment. They thank all pregnant women for agreeing to participate in the study.

\section{Disclosure}

The authors report no conflicts of interest in this work.

\section{References}

1. De Beaudrap P, Turyakira E, White LJ, et al. Impact of malaria during pregnancy on pregnancy outcomes in a Ugandan prospective cohort with intensive malaria screening and prompt treatment. Malar J. 2013;12:139.

2. Dreyfuss ML, Msamanga GI, Spiegelman D, et al. Determinants of low birth weight among HIV-infected pregnant women in Tanzania. Am J Clin Nutr. 2001;74(6):814-826.

3. WHO. Malaria and HIV interactions and their implications for public health policy. Conclusions of a technical consultation convened by WHO. Geneva: WHO; 2004. Available from: http://www.who.int/hiv/ pub/prev_care/malariahiv.pdf. Accessed July 20, 2016.

4. Uneke CJ, Ogbonna A. Malaria and HIV co-infection in pregnancy in sub-Saharan Africa: impact of treatment using antimalarial and antiretroviral agents. Trans R Soc Trop Med Hyg. 2009;103(8):761-767.

5. Radeva-Petrova D, Kayentao K, ter Kuile FO, Sinclair D, Garner P. Drugs for preventing malaria in pregnant women in endemic areas: any drug regimen versus placebo or no treatment. Cochrane Database Syst Rev. 2014;(10):CD000169.

6. Tanzania National Bureau of Statistics. Tanzania HIV/AIDS and Malaria Indicator Survey 2007-2008. Available from: http://www.nbs.go.tz/ nbstz/index.php/english/statistics-by-subject/health-statistics/hiv-andmalaria-survey/679-2007-08-tanzania-hiv-aids-and-malaria-indicatorsurvey-thmis-report. Accessed October 13, 2016.

7. Tanzania National Bureau of Statistics. Tanzania HIV/AIDS and Malaria Indicator Survey 2011-2012. Available from: http://www.nbs.go.tz tnada/index.php/catalog/23. Accessed October 13, 2016.

8. Tanzania National Bureau of Statistics. Tanzania Demographic Health Survey and Malaria Indicator Survey 2015-2016. Available from: http://www.nbs. go.tz/nbstz/index.php/english/statistics-by-subject/health-statistics/demographic-and-health-survey-dhs/730-the-2015-16-tanzania-demographicand-health-survey-and-malaria-indicator-survey-key-findings-report. Accessed October 13, 2016.

9. Ministry of Health and Social Welfare. Tanzania PMTCT report. Available from: http://pmtct.or.tz/pmtct-tanzania/pmtct-in-tanzania. Accessed October 9, 2016.

10. UNAIDS. Global report. 2013. Available from: http://www.unaids.org/ sites/default/files/media_asset/UNAIDS_Global_Report_2013_en_1. pdf. Accessed October 9, 2016.

11. Iliyasu Z, Babashani M, Abubakar IS, Salahudeen AA, Aliyu MH. Clinical burden and correlates of HIV and malaria co-infection, in northwest Nigeria. Acta Trop. 2013;128(3):630-635.

12. Mother to Child Transmission (MTCT). Center for AIDS Prevention Studies. Prevention Research Centre. UCSF. Is mother-to-child HIV transmission preventable? Available from: http://caps.ucsf.edu/archives/ factsheets/mother-to-child-transmission-mtct. Accessed October 9, 2016

13. Mutagonda R, Kamuhabwa AR, Massawe S, Mpembeni R. Intermittent preventive therapy and treatment of malaria during pregnancy: a study of knowledge among pregnant women in Rufiji District, Southern Tanzania. Trop J Pharm Res. 2012;11:835-845.

14. WHO. Consolidated guidelines on the use of antiretroviral drugs for treating and preventing HIV infection. Recommendations for a public health approach. Geneva: WHO; 2013. Available from: http://apps. who.int/iris/bitstream/10665/85321/1/9789241505727_eng.pdf?ua=1. Accessed August 4, 2016.

15. Suthar AB, Vitoria MA, Nagata JM, et al. Co-trimoxazole prophylaxis in adults, including pregnant women, with HIV: a systematic review and meta-analysis. Lancet HIV. 2015;2(4):e137-e150.
16. Ward SA, Sevene EJ, Hastings IM, Nosten F, McGready R. Antimalarial drugs and pregnancy: safety, pharmacokinetics, and pharmacovigilance. Lancet Infect Dis. 2007;7(2):136-144.

17. Sevene E, González R, Menéndez C. Current knowledge and challenges of antimalarial drugs for treatment and prevention in pregnancy. Expert Opin Pharmacother. 2010;11(8):1277-1293.

18. Peters PJ, Thigpen MC, Parise ME, Newman RD. Safety and toxicity of sulfadoxine/pyrimethamine: implications for malaria prevention in pregnancy using intermittent preventive treatment. Drug Saf. 2007;30(6):481-501.

19. Ministry of Health and Social Welfare. Tanzania National Guidelines for the Management of HIV and AIDS. National AIDS Control Programme. Fourth Edition (April 2012). Available from: www.nacp.go.tz/ site/download/nationalguideline42012.pdf. Accessed July 25, 2016.

20. Kamuhabwa AAR, Silumbe R. Knowledge among drug dispensers and antimalarial drug prescribing practices in public health facilities in Dar es Salaam. Drug Healthc Patient Saf. 2013;5:181-189.

21. Kamuhabwa AA, Manyanga V. Challenges facing effective implementation of co-trimoxazole prophylaxis in children born to HIV-infected mothers in the public health facilities. Drug Healthc Patient Saf. 2015;7:147-156.

22. Focused antenatal care in Tanzania. Country-level programmes. Available from: https://cdn2.sph.harvard.edu/wp-content/uploads/ sites/32/2014/09/HSPH-Tanzania5.pdf. Accessed October 9, 2016.

23. Manyahi J, Jullu BS, Abuya MI, et al. Prevalence of HIV and syphilis infections among pregnant women attending antenatal clinics in Tanzania, 2011. BMC Public Health. 2015;15:501.

24. WHO. Guidelines on co-trimoxazole prophylaxis for HIV-related infections among children, adolescents, and adults. Recommendations for a public health approach. Geneva: WHO; 2006. Available from: http:// www.who.int/hiv/pub/guidelines/ctxguidelines.pdf. Accessed July 25, 2016.

25. Kapito-Tembo A, Meshnick SR, van Hensbroek MB, Phiri K, Fitzgerald M, Mwapasa V. Marked reduction in prevalence of malaria parasitemia and anemia in HIV-infected pregnant women taking co-trimoxazole with or without sulfadoxine-pyrimethamine intermittent preventive therapy during pregnancy in Malawi. J Infect Dis. 2011;203(4):464-472.

26. Tudor Car L, van-Velthoven MH, Brusamento S, et al. Integrating prevention of mother-to-child HIV transmission (PMTCT) programmes with other health services for preventing HIV infection and improving HIV outcomes in developing countries. Cochrane Database Syst Rev. 2011;(6):CD008741.

27. Baker U, Okuga M, Waiswa P, Manzi F, Peterson S, Hanson C; EQUIP Study Group. Bottlenecks in the implementation of essential screening tests in antenatal care: syphilis, HIV, and anemia testing in rural Tanzania and Uganda. Int J Gynaecol Obstet. 2015;130 Suppl 1:S43-S50.

28. Manyanga VP, Minzi O, Ngasala B. Prevalence of malaria and anaemia among HIV infected pregnant women receiving co-trimoxazole prophylaxis in Tanzania: a cross sectional study in Kinondoni Municipality. BMC Pharmacol Toxicol. 2014;15:24.

29. Mwambete KD, Kamuhabwa AAR. Resistance of commensal intestinal Escherichia coli and other enterics to co-trimoxazole and commonly used antibiotics in HIV/AIDS patients. Clin Microbiol. 2013;3:134.

30. WHO Library Cataloguing-in-Publication Data. Co-trimoxazole prophylaxis for HIV-exposed and HIV-infected infants and children: practical approaches to implementation and scale up. Available from: http://www.who.int/hiv/pub/tuapr_2009_en.pdf. Accessed November 8, 2016.

31. Ford N, Shubber Z, Jao J, Abrams JE, Frigati L, Mofenson L. Safety of co-trimoxazole in pregnancy: a systematic review and meta-analysis. J Acquir Immune Defic Syndr. 2014;66(5):512-521.

32. Aizire J, Fowler MG, Wang J, et al. Extended prophylaxis with nevirapine and cotrimoxazole among HIV-exposed uninfected infants is well tolerated. AIDS. 2012;26(3):325-333.

33. Ivan E, Crowther NJ, Mutimura E, Osuwat LO, Janssen S, Grobusch MP. Helminthic infections rates and malaria in HIV-infected pregnant women on anti-retroviral therapy in Rwanda. PLoS Negl Trop Dis. 2013;7(8):e2380. 
34. Jimmy B, Jose J. Patient medication adherence: measures in daily practice. Oman Med J. 2011;26(3):155-159.

35. Pell C, Meñaca A, Were F, et al. Factors affecting antenatal care attendance: results from qualitative studies in Ghana, Kenya and Malawi. PLoS One. 2013;8(1):e53747.

36. Mutagonda R, Kamuhabwa A, Massawe S. Intermittent preventive therapy and treatment of malaria during pregnancy: perspectives of health care providers in Rufiji District, Southern Tanzania. East Afr J Public Health. 2014;11(2):680-694.
37. Mboera LE, Rumisha SF, Senkoro KP, Mayala BK, Shayo EH, Kisinza WN. Knowledge and health information communication in Tanzania. East Afr J Public Health. 2007;4(1):33-39.

38. Silumbe S, Kamuhabwa AAR. Management of antimalarial drugs in the urban public health facilities in Tanzania. Int J Pharm Sci Res. 2015;6(1):154-163.

39. Clark M, Barraclough N. Managing medicines and health products. In: Health Systems in Action: An E-Handbook for Leaders and Managers. USAID/MSH. Medford (MA): Management Sciences for Health; 2010.

\section{Publish your work in this journal}

Drug, Healthcare and Patient Safety is an international, peer-reviewed open access journal exploring patient safety issues in the healthcare continuum from diagnostic and screening interventions through to treatment, drug therapy and surgery. The journal is characterized by the rapid reporting of reviews, original research, clinical, epidemiological and post-marketing surveillance studies, risk management, health literacy and educational programs across all areas of healthcare delivery. The manuscript management system is completely online and includes a very quick and fair peer-review system. Visit http://www.dovepress.com/ testimonials.php to read real quotes from published authors. 